\title{
Mechanical and biomechanical analysis of a linear piston design for angular-velocity-based orthotic control
}

\author{
Edward D. Lemaire, PhD; ${ }^{1-2 *}$ Reza Samadi, MASc; ${ }^{1}$ Louis Goudreau, PEng; ${ }^{1}$ Jonathan Kofman, PhD, PEng ${ }^{3}$ \\ ${ }^{1}$ Ottawa Hospital Research Institute, The Ottawa Hospital Rehabilitation Centre, Ottawa, Canada; ${ }^{2}$ Faculty of Medicine, \\ University of Ottawa, Ottawa, Canada; ${ }^{3}$ Department of Systems Design Engineering, University of Waterloo, Waterloo, \\ Canada
}

\begin{abstract}
A linear piston hydraulic angular-velocity-based control knee joint was designed for people with knee-extensor weakness to engage knee-flexion resistance when knee-flexion angular velocity reaches a preset threshold, such as during a stumble, but to otherwise allow free knee motion. During mechanical testing at the lowest angular-velocity threshold, the device engaged within 2 degrees knee flexion and resisted moment loads of over $150 \mathrm{Nm}$. The device completed 400,000 loading cycles without mechanical failure or wear that would affect function. Gait patterns of nondisabled participants were similar to normal at walking speeds that produced belowthreshold knee angular velocities. Fast walking speeds, employed purposely to attain the angular-velocity threshold and cause knee-flexion resistance, reduced maximum knee flexion by approximately 25 degrees but did not lead to unsafe gait patterns in foot ground clearance during swing. In knee collapse tests, the device successfully engaged knee-flexion resistance and stopped knee flexion with peak knee moments of up to $235.6 \mathrm{Nm}$. The outcomes from this study support the potential for the linear piston hydraulic knee joint in knee and kneeankle-foot orthoses for people with lower-limb weakness.
\end{abstract}

Key words: angular velocity, angular-velocity-based orthosis, cyclic testing, gait, knee-ankle-foot orthosis, mechanical testing, orthosis, Ottawalk-Speed, stance control, stance-control orthosis.

\section{INTRODUCTION}

Stance-control orthoses (SCOs) are lower-limb assistive devices that prevent knee collapse during weightbearing while allowing free knee motion during the swing phase of gait [1-18]. The methods of controlling and applying knee-flexion resistance, device complexity, device function, and user acceptance vary considerably between stance-control devices. Many SCOs use the precondition of weight-bearing to engage knee-flexion resistance, with or without a specific lower-limb segment orientation or joint-angle requirement. Mechanical or electromechanical methods are commonly used to detect these preconditions. The devices then apply knee-flexion resistance as appropriate. Unfortunately, the method of applying knee-flexion resistance and the methods of detecting weight-bearing, limb-segment orientation, and

\footnotetext{
Abbreviations: $\mathrm{AVBC}=$ angular-velocity-based control, $\mathrm{KAFO}=$ knee-ankle-foot orthosis, OWS = Ottawalk-Speed, SCKAFO = stance-control knee-ankle-foot orthosis, SCO = stance-control orthosis.

*Address all correspondence to Edward D. Lemaire, PhD; The Ottawa Hospital Rehabilitation Centre, 505 Smyth Rd, Ottawa, ON, Canada K1H 8M2; 613-737-7350, ext 75592. Email: elemaire@toh.on.ca

http://dx.doi.org/10.1682/JRRD.2012.02.0031
} 
joint-angle preconditions may contribute to a complex, bulky, and costly mechanical or electromechanical system. This is especially true if the detection of the precondition and the application of knee-flexion resistance are performed by separate means.

Mechanical stance-control knee-ankle-foot orthoses (SCKAFOs) either require full knee extension to lock and unlock the knee or resist knee flexion at any angle. Orthoses that require full knee extension typically use a spring-loaded or weighted pawl to lock the knee in extension when the leg is in a preset position at foot strike [1213]. While these devices are smaller and lighter than devices that provide resistance at any knee angle, the orthosis cannot stop leg collapse when the person lands with a flexed knee, such as during a stumble or during curb or step navigation.

Mechanical SCKAFOs that resist flexion at any knee angle require a mechanism at the foot to determine when the user is weight-bearing [14-16]. These mechanisms include a double-shell ankle-foot orthosis that pushes up on a control rod during weight-bearing, a hinged footplate that engages knee-flexion resistance based on ankle angle, or a pneumatic bladder that engages upon weight-bearing.

SCKAFOs with electronic control systems resist knee flexion at any knee angle. Plantar pressure sensors, sometimes combined with position sensors, sense weightbearing and provide context-based decision-making to control knee-flexion resistance [6-7,11,14,17]. Control electronics and batteries are either housed on the orthoses or in a belt pack. Although electronic control systems can improve stance swing and activity-based mode switching reliability, they add weight, complexity, and power management requirements to the orthotic device.

One mechanical method that did not require limbloading detection used a knee-joint spring to provide an extension moment that decreased as the moment arm shortened during knee flexion [18]. Since the knee extension moment dropped to zero for swing and sitting, knee angle-based stance control was achieved. The orthosis was designed to not resist knee flexion beyond a preset knee angle; however, resistance at any knee angle may be required for stance control in stumbling. Incorporating knee-angle detection inherently in the knee flexionresistance mechanism, with the spring line of action changing with angle, was a desired feature for minimizing device complexity, weight, bulk, and cost.

Another orthosis used inertial, angular-position, and force sensors in a custom actuator system to apply vary- ing stiffness to an orthosis knee joint [10]. Since the system used predetermined relationships between resisting torque and joint angle to switch between stance and swing modes, the device may not handle unexpected conditions such as stumbling.

An angular-velocity-based control (AVBC) approach differs from weight-bearing, limb-segment orientation, and joint angle-based control devices by removing the need for mechanical systems or electronic sensors at the foot to maintain safe gait for people with knee-extensor weakness but sufficient hip control [19]. The AVBC approach is based on the premise that knee-flexion angular velocity during limb collapse is greater than during walking. With a hydraulic implementation of this approach, fluid flow increases as the knee angular velocity increases. The resulting increase in fluid-generated forces on a control valve, set for a predetermined angular-velocity threshold, causes the valve to close when knee angular velocity reaches the preset value, providing flexion resistance in unexpected limb-orientation and limb-loading conditions such as during a stumble.

In a previous design, a rotary hydraulic approach was used to provide knee-flexion resistance during leg collapse but allow free knee motion during walking [19]. The AVBC joint was a self-contained unit with angular velocity sensing, activation, and application of hydraulic flexion resistance all located within the orthotic knee joint, enabling use in SCKAFOs as well as knee orthoses. While this approach successfully handled the load capacity for a $90 \mathrm{~kg}$ user, medial and lateral joints were required to reliably handle leg-collapse events. Also, during a legcollapse event, knee-flexion resistance engaged at approximately $40^{\circ}$ knee flexion, stopped knee flexion, and then continued to slow flexion by an additional $12^{\circ}$ over the next $2 \mathrm{~s}$ while the user recovered. Improvements were recommended to have the knee joint engage knee-flexion resistance within a smaller range, limit knee flexion after engagement, and optimize device size and weight.

This article presents the mechanical testing and biomechanical evaluation of a linear piston hydraulic design for angular-velocity-based orthotic knee-flexion control. The device was designed to resist leg collapse at the knee under high load while maintaining acceptable orthosis size and weight. This device improves on the previous rotary design by reducing time to knee-joint engagement, knee flexion after engagement, and device size and weight.

The angular-velocity-based SCO would be used by people who have isolated knee extensor weakness but can 
function with a free motion knee joint for most activities (i.e., able to consistently extend the knee and thereby support themselves during the weight-bearing phase of gait). Examples are people with femoral neuropathy or polio. A portion of the knee-ankle-foot orthosis (KAFO) user population would still require an orthosis that rigidly controls knee flexion during every step for confident and safe locomotion.

\section{METHODS}

\section{Design}

The angular-velocity-based SCO, termed the OttawalkSpeed (OWS), was designed to have the entire device enclosed in a compact knee-joint unit and meet the following objectives: engage knee-flexion resistance at a predetermined knee-flexion angular velocity threshold, permit free knee motion when not engaged, provide adequate kneeflexion resistance using only a single device located on the lateral side with a standard single-axis joint on the medial side, allow easy integration with standard orthotic components, permit easy adjustment of the threshold angular velocity required for engagement, have minimal frontalplane dimensions to maintain cosmesis, limit the likelihood of medial joint contact, and function quietly.

We used a novel linear piston design to achieve the design objectives (Figure 1). The linear piston was better able to accommodate higher pressures while providing unrestricted motion with an open valve than the earlier rotary piston design, with similar size and weight [19]. We used a standard interface to attach upper and lower orthotic uprights to the hydraulic knee joint. The hydraulic knee joint utilized a slider-crank mechanism design with an (1) upper-upright and hydraulic-cylinder frame, (2) lowerupright crank, (3) connecting-rod link, and (4) piston slider (Figure 1). The upper-upright frame, to which the hydraulic cylinder was fixed, was pin-connected to the lowerupright crank at the knee-joint axis. One novel feature of the linear piston design was to have the connecting-rod link bear on the upper-upright frame to support the force component normal to the piston, thereby reducing the force that would be transmitted to the piston. A custom-made adjustable ball-based check valve was attached to the end of the cylinder and a fluid reservoir was attached to the valve.

The linear hydraulic design's valve principle was the same as the rotary design [19]. As the knee flexes, the piston pushes fluid through a spring-biased valve. The flow

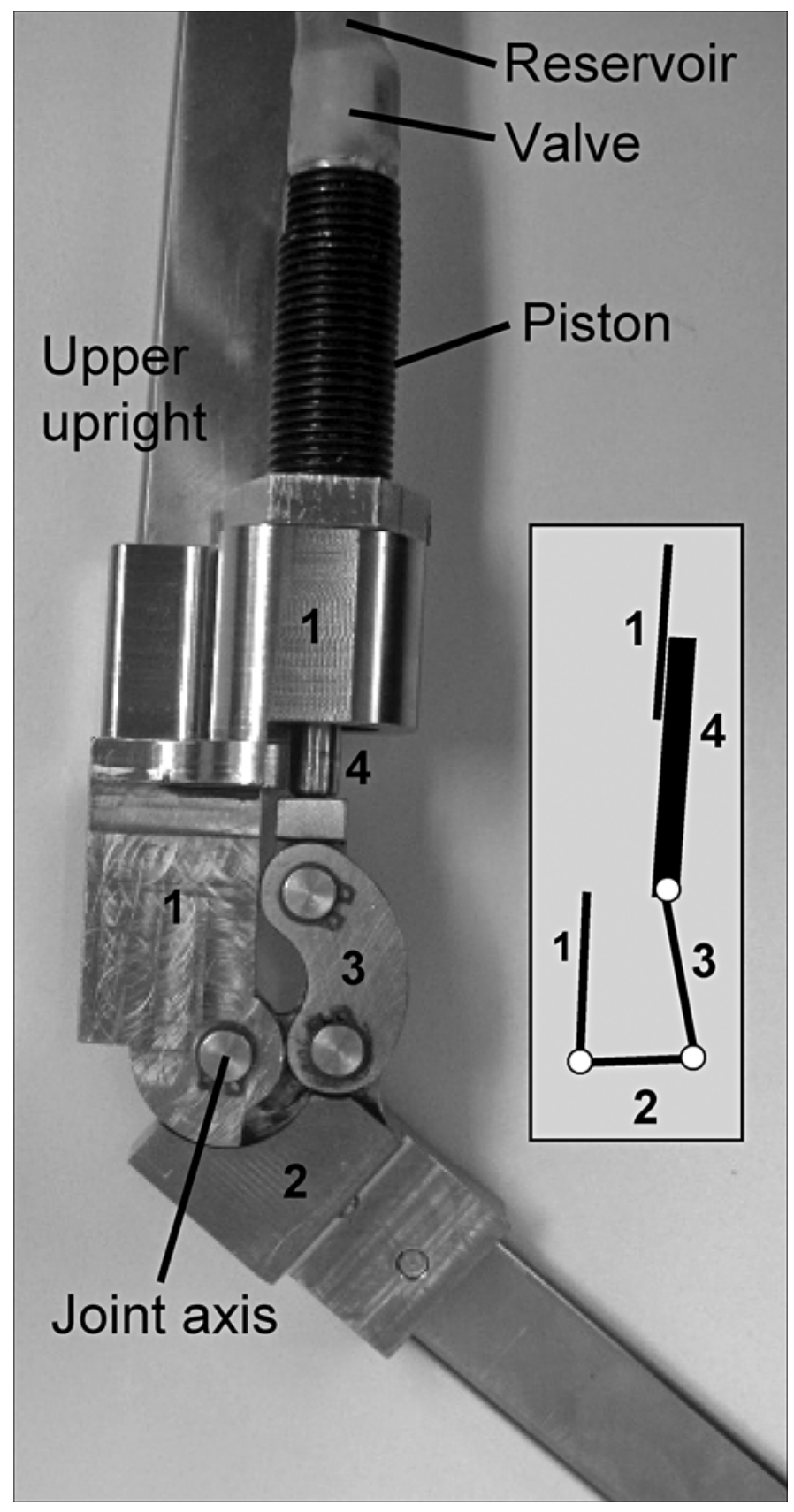

Figure 1.

Ottawalk-Speed linear piston hydraulic knee joint showing slider-crank mechanism illustrated in inset diagram: (1) upperupright and hydraulic-cylinder frame, (2) lower-upright crank, (3) connecting-rod link, and (4) piston slider. (1) Upper-upright and hydraulic-cylinder frame is pin-connected to (2) lowerupright crank at knee-joint axis.

rate produces a drag force on the valve's ball proportional to the square of the fluid velocity and the projected area of 
the valve perpendicular to the direction of fluid flow. As knee angular velocity increases, the drag force on the ball can surpass the spring force, thereby moving the ball into the valve channel to stop fluid flow from the piston to the fluid reservoir. The resulting increase in pressure stops further knee flexion. When the knee extends, pressure is removed and the spring pushes the ball away from the valve channel and free knee motion resumes. Adjusting the effective spring length changes the drag-force threshold at which the valve closes and consequently the angular velocity at which knee-flexion resistance engages.

We found commercially available hydraulic cylinders to be appropriate for the OWS requirements (hydraulic threaded mini-cylinders, Vektek Inc; Emporia, Kansas). The overall dimensions were $190.00 \times 39.30 \times 29.14 \mathrm{~mm}$. We modified the hydraulic cylinder to remove unused space that, together with a reservoir optimized for length, resulted in a device length $<170 \mathrm{~mm}$. The main joint structure was similar in size to a standard orthotic knee joint (approximately $100 \mathrm{~mm}$ in length).

\section{Mechanical Tests}

We fabricated an OWS hydraulic stance-control knee joint for mechanical testing. We completed dynamic loading and cyclic loading tests to evaluate the mechanical behavior and resistance to failure of the hydraulic orthotic knee joint, respectively.

We performed load tests on the hydraulic knee joint using a material testing machine (model 4482, Instron Industrial Products; Grove City, Pennsylvania) to determine passive joint flexion resistance, time to engagement of the resistance, maximum joint flexion resistance, and the corresponding flexion angular velocities.

The passive movement test evaluated the residual resistance to joint angular motion when knee-flexion resistance is not engaged. The valve closing threshold was set above the angular velocity that corresponded to the testing machine's maximum loading rate $(500 \mathrm{~mm} / \mathrm{min}$ crosshead travel speed) to ensure that flexion resistance did not engage during testing. We conducted four loading trials at the $500 \mathrm{~mm} / \mathrm{min}$ rate.

The knee-flexion resistance evaluation involved setting the valve to the lowest valve closing angular-velocity threshold to ensure that flexion resistance engaged during testing and loading the joint at the maximum testingmachine rate until a maximum joint resisting moment of approximately $150 \mathrm{Nm}$ was reached. We based this value on stair ascent knee moments for a $90 \mathrm{~kg}$ user [14]. We recorded 20 trials.

We performed cyclic loading tests on the knee joint using a custom testing machine that cyclically loaded the joint to a $50 \mathrm{Nm}$ resistance moment every $2 \mathrm{~s}$. This load is consistent with knee-flexion loading during walking for a $90 \mathrm{~kg}$ user. The joint was set to engage at the lowest threshold angular velocity to ensure engagement at every cycle. In testing future prototypes, a faster loading rate that replicates anticipated loading patterns could be considered. We tested the current prototype joint to a maximum of 400,000 cycles, since flexion resistance would typically not activate for each step during walking but only during stumbling or other fast knee-flexion events. This number of cycles is a conservative usage estimate that would permit more than 1,000 joint activations per day over 1 yr.

\section{Biomechanical Tests}

We recruited five nondisabled male participants from The Ottawa Hospital Rehabilitation Centre (age [mean \pm standard deviation] $=36.0 \pm 8.5 \mathrm{yr}$, weight $=78.9 \pm 11.4 \mathrm{~kg}$, height $=178.6 \pm 2.2 \mathrm{~cm}$ ) for biomechanical testing of the OWS orthosis. Nondisabled participants were warranted since (1) some uncertainty existed for flexion-resistance performance during full weight-bearing; (2) loads imparted on the joint from nondisabled gait are sufficient for preliminary analysis of joint function; (3) analyses would show how the orthosis affects normal gait, whereas the variety of gait deviations in pathological gait would be difficult to control or compensate for in the analysis; and (4) collapse events could be carried out in a controlled manner.

A certified orthotist fit the participants with an OWS SCKAFO. The orthosis employed the OWS hydraulic joint on the lateral side and a standard uniaxial joint on the medial side and the polypropylene ankle-foot component used Tamarack Habilitation Technologies (Blaine, Minnesota) ankle joints. The orthotist qualitatively adjusted the OWS for each participant such that flexion resistance did not engage at a self-selected walking speed.

\section{Data Collection}

We performed all data collection trials in the Rehabilitation Technology Laboratory at The Ottawa Hospital Rehabilitation Centre. We collected lower-limb marker position and ground-reaction force data at $100 \mathrm{~Hz}$ using a sevencamera motion capture system (Vicon Motion Systems; Los Angeles, California), Vicon Nexus software (Vicon Motion Systems), six degree-of-freedom marker set, and force 
plate (AMTI Force and Motion; Watertown, Massachusetts). We imported all marker and ground-reaction force data into Visual3D software (C-Motion Inc; Germantown, Maryland) to calculate joint kinematics and kinetics. We exported all kinematic and kinetic data into Excel (Microsoft; Redmond, Washington) for post-processing.

Once we affixed the reflective markers to the participant and obtained the required anthropometric data, the participant walked through a $6 \mathrm{~m}$-long data-capture volume. Participants performed five trials walking at a selfselected pace that maintained knee angular velocity below the knee-resistance engagement threshold. We captured bilateral knee kinematic data during each trial.

Subsequently, the participants performed five trials walking at a faster pace that purposely allowed the knee angular velocity to reach the threshold for knee-resistance engagement. This test was important since knee-flexion resistance during swing would result in reduced foot clearance and possibly result in toe contact during swing and risk of a stumble or fall. We captured bilateral knee kinematic data during each trial.

Participants performed five further trials standing with the braced leg on a force platform. With a spotter nearby, the participant let the braced leg collapse such that all weight was transferred to the braced leg, and then the participant recovered body position to stationary standing. Participants relaxed their leg muscles as much as possible during collapse, with a series of practice trials performed before testing to give participants confidence that the orthosis would prevent a fall. We intended this test to represent the worst-case stumbling scenario, where users would rapidly transfer all of their body weight to the braced leg. Knee collapse cycles started with the initiation of braced-leg collapse, where body weight was transferred onto the braced limb, and ended with the contralateral foot touching the floor. We captured kinematic and kinetic data from the braced leg during each trial.

\section{RESULTS}

\section{Mechanical Tests}

For the passive motion test with the valve open, the average resistance to motion was $1.07 \pm 0.24 \mathrm{Nm}$, determined from forces measured by the material testing machine, and is negligible for human leg movement. The pin joints for the testing machine fixtures may have con- tributed to this resistance, since the fixtures were not optimized for frictionless motion. Therefore, the joint likely had an even lower resistance to motion. Based on qualitative feedback, orthotists considered the low level of resistance to be acceptable.

For the knee-flexion resistance evaluation with the valve closed, flexion resistance was activated within $2^{\circ}$ over a period of $0.55 \mathrm{~s}$ from initiation of motion in all trials. These results are a substantial improvement over the rotary piston prototype [19]. The joint successfully supported loads of up to $153.1 \mathrm{Nm}$, with an average of $144.6 \pm$ 4.9 Nm over the 20 trials.

The OWS successfully completed 400,000 cycles of repeated loading at the target load. Based on visual inspection, we encountered no evidence of mechanical or hydraulic failure. The OWS performed as expected when mounted in a KAFO following the cyclic testing.

\section{Biomechanical Tests}

At walking speeds that maintained knee angular velocity below the threshold for knee-resistance engagement, the orthosis had minimal effect on knee kinematics, as desired. During fast walking (threshold-reached) trials, the maximum knee angle was reduced on the braced limb (Figure 2). Braced-limb knee angle was greater during stance for fast walking trials than in the below-threshold trials. During swing, the peak knee angle at 65 to 75 percent gait cycle was lower in the fast walking trials than in the below-threshold trials (Figure 2) due to knee-flexion resistance engagement, as expected. Table 1 shows that mean braced-limb knee angles were lower for the fast walking trials than for the below-threshold trials. Bracedlimb knee angular velocity and walking speed were greater for the fast walking than the below-threshold trials (Table 1), except for peak angular velocity during swing (85\%-95\% gait cycle, Figure 2). Pelvic obliquity angles were similar for both walking speeds (Figure 3).

For leg-collapse trials, the mean knee angular velocity for all participants increased to a maximum of $208.7 \pm$ $51.9 \%$ s. Flexion resistance engaged at this maximum angular velocity. Subsequently, the knee-resistance moment increased and angular velocity decreased over a range of $20.9^{\circ} \pm 6.3^{\circ}$ until knee flexion stopped. The average time between flexion resistance engaging and knee flexion stopping was $0.20 \pm 0.04 \mathrm{~s}$.

During leg collapse for participant 2 (Figure 4), knee flexion resistance engaged at an average of 16 percent of the cycle $\left(34.5^{\circ}\right)$ and knee flexion stopped at 32 percent 

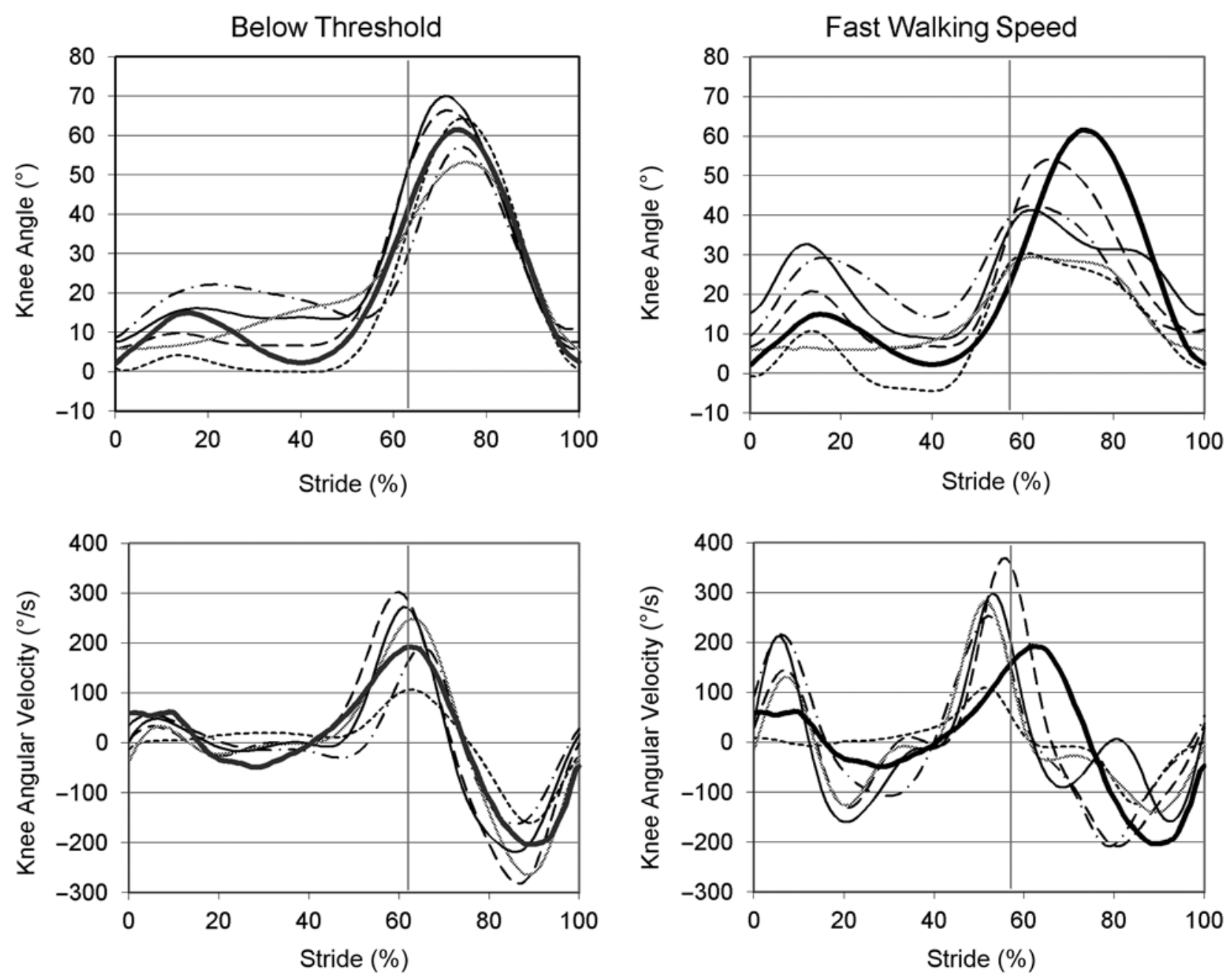

Figure 2.

Mean (over 5 trials) knee angle (top row) and knee angular velocity (bottom row) of braced limb for five subjects at below-threshold (left column) and fast walking speeds (right column) that enabled threshold angular velocities. Nondisabled normal walking kneeangle and angular-velocity curves shown in bold for comparison.

$\left(61.3^{\circ}\right)$, with the mean maximum knee moment of $175 \mathrm{Nm}$ occurring at 29 percent of the cycle.

For all subjects during leg collapse, the average knee moment provided by the OWS increased from knee-flexion resistance engagement to a maximum of $1.9 \pm 0.73 \mathrm{Nm} / \mathrm{kg}$, when knee flexion stopped. The knee remained at approximately the same flexion angle $\left(43.5^{\circ} \pm 10.6^{\circ}\right)$ for the remainder of the cycle. During this support phase, the knee moment averaged $0.54 \pm 0.33 \mathrm{Nm} / \mathrm{kg}$. One participant had a maximum knee moment of $235.6 \mathrm{Nm}$.

Table 2 compares the linear and rotary hydraulic designs' dimensions, weight, and performance during similar knee collapse testing trials [19].

\section{DISCUSSION}

An orthotic device that can selectively resist knee flexion at any knee angle but does not require limbloading information to determine when to engage flexion resistance would fill a gap in the assistive technology options available for people with knee extensor weakness. The outcomes from this study demonstrated that the AVBC approach implemented with the linear hydraulic design is a viable solution.

Mechanical evaluation of the OWS showed that only one lateral flexion-control joint would be required to accommodate the anticipated loads during knee collapse 
events for a $90 \mathrm{~kg}$ user. Since the device had minimal resistance to passive motion, minimal effort would be required for people with lower-limb weakness to initiate knee movement. The OWS activated flexion resistance upon reaching the preset angular-velocity threshold within only $2^{\circ}$ of joint flexion. Rapid engagement is important to ensure safety and user confidence in the assistive device.

No wear was visually present after completing 400,000 cycles of repetitive joint loading. For a prototype device on humans, 400,000 cycles is an appropriate target since the

Table 1.

Mean \pm standard deviations of knee angle, knee angular velocity, and walking speed for five participants by condition (braced- and unbraced-limb and below-threshold and threshold-reached).

\begin{tabular}{lccccc}
\hline \multicolumn{1}{c}{ Condition } & Participant 1 & Participant 2 & Participant 3 & Participant 4 & Participant 5 \\
\hline Below-Threshold & & & & & \\
Braced Knee Angle $\left({ }^{\circ}\right)$ & $-70.3 \pm 1.8$ & $-66.5 \pm 3.5$ & $-53.7 \pm 2.7$ & $-57.4 \pm 3.2$ & $-65.2 \pm 1.6$ \\
Unbraced Knee Angle $\left({ }^{\circ}\right)$ & $-64.5 \pm 0.9$ & $-59.9 \pm 2.7$ & $-45.1 \pm 4.2$ & $-48.3 \pm 25.2$ & $-63.2 \pm 1.1$ \\
Braced Knee Angular Velocity ( $\%$ s) & $274.3 \pm 20.9$ & $303.6 \pm 9.8$ & $106.4 \pm 11.6$ & $194.0 \pm 24.0$ & $268.0 \pm 23.1$ \\
Unbraced Knee Angular Velocity (\%/s) & $253.6 \pm 5.8$ & $263.8 \pm 11.7$ & $120.4 \pm 2.5$ & $177.2 \pm 26.3$ & $219.3 \pm 21.8$ \\
Walking Speed (m/s) & $0.76 \pm 0.03$ & $0.98 \pm 0.03$ & $0.53 \pm 0.02$ & $0.59 \pm 0.03$ & $0.68 \pm 0.11$ \\
Threshold-Reached & & & & \\
Braced Knee Angle $\left({ }^{\circ}\right)$ & $-41.5 \pm 0.3$ & $-54.3 \pm 1.7$ & $-29.5 \pm 2.6$ & $-42.7 \pm 0.9$ & $-30.7 \pm 1.1$ \\
Unbraced Knee Angle $\left({ }^{\circ}\right)$ & $-55.1 \pm 30.9$ & $-63.2 \pm 2.0$ & $-41.1 \pm 23.1$ & $-51.1 \pm 1.3$ & $-72.2 \pm 1.5$ \\
Braced Knee Angular Velocity (\%/s) & $299.0 \pm 24.4$ & $377.7 \pm 12.2$ & $118.7 \pm 5.4$ & $263.2 \pm 29.2$ & $291.4 \pm 10.0$ \\
Unbraced Knee Angular Velocity ( $\% / \mathrm{s})$ & $334.2 \pm 187.8$ & $344.1 \pm 7.3$ & $183.9 \pm 105.2$ & $357.2 \pm 20.7$ & $370.0 \pm 15.2$ \\
Walking Speed (m/s) & $1.49 \pm 0.05$ & $1.41 \pm 0.13$ & $0.98 \pm 0.08$ & $1.62 \pm 0.02$ & $1.45 \pm 0.09$ \\
\hline \hline
\end{tabular}

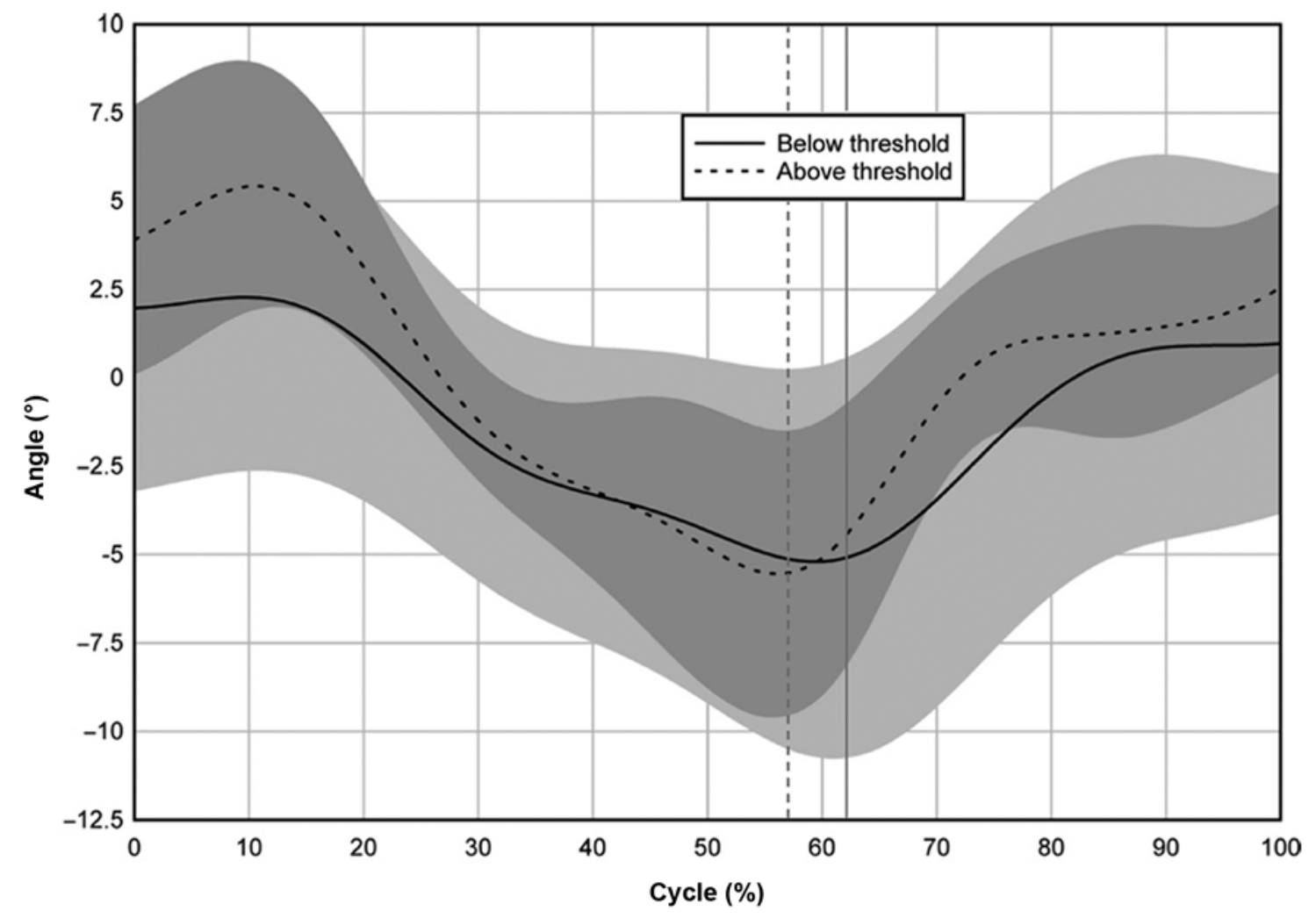

Figure 3.

Mean (over 5 trials) pelvic obliquity angles for below-threshold and fast walking speeds. Light band represents below-threshold standard deviations and dark band represents fast walking speed standard deviations. Vertical lines represent average toe-off instant. 


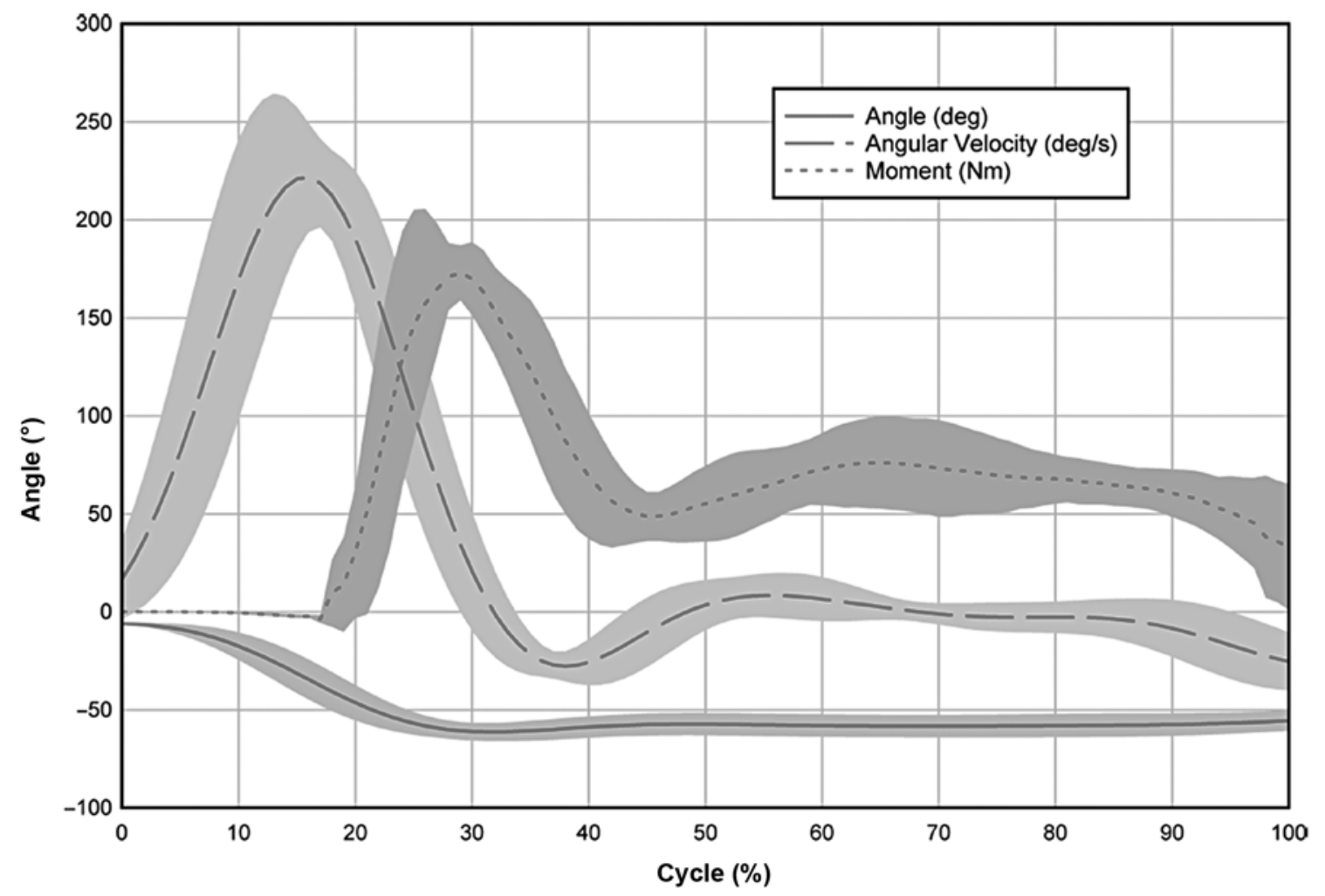

Figure 4.

Mean braced-limb knee angle, angular velocity, and moment during leg collapse for participant 2. Shaded regions show standard deviations.

Table 2.

Comparison between linear and rotary hydraulic designs for angularvelocity control.

\begin{tabular}{lcc}
\hline \multicolumn{1}{c}{ Comparison } & Linear & Rotary \\
\hline $\begin{array}{l}\text { Time to Knee-Joint Engagement } \\
\quad \text { During Collapse (s) }\end{array}$ & 0.20 & 0.27 \\
Knee Flexion After Engagement $\left(^{\circ}\right)$ & 2 & 12 \\
$\begin{array}{l}\text { Device Length } \times \text { Width } \times \\
\quad \text { Thickness }(\mathrm{mm})\end{array}$ & $190 \times 39 \times 29$ & $140 \times 67 \times 30$ \\
Device Weight $(\mathrm{g})$ & 394 & $396 \times 2$ joints \\
\hline \hline
\end{tabular}

joint would not normally reach the angular-velocity threshold and engage flexion resistance for every step in walking.

The orthotic device performed as expected for walking and knee collapse trials. For below-threshold walking, knee angle curves were similar in shape to normal gait for all participants, with minor deviations in the magnitude of knee flexion during swing and stance flexion. These deviations could be attributable to the wearing of a single-axis KAFO and the limited device accommodation time for the nondisabled participants.
To test for the effect of flexion resistance engaging during walking, participants walked at a speed fast enough to purposely engage the OWS. In these cases, braced-limb knee flexion was reduced during swing by an average of 28 percent compared with the unbraced limb. For most participants, the knee-angle curve was truncated (i.e., lower peak value and/or flatter shape) during swing, with the appropriate angular gait pattern resuming at terminal swing. A knee-flexion reduction at the initiation of swing could have potentially introduced foot-clearance issues; favorably, we found no issues during walking. Even with minimal OWS experience, participants were able to clear the braced limb during swing when walking at speeds that produced angular-velocity control without excessive accommodation movements or training. Therefore, end users should not be at risk if they speed up their walking gait periodically. While this result is encouraging, a training regime for people with knee-extension weakness should include instruction on potential toe-ground contact during the initial swing phase if the person walks excessively fast. An 
accommodated gait strategy may be appropriate for these short periods of excessively fast walking.

The leg-collapse trials demonstrated the viability of the OWS to stop knee flexion and maintain the knee support angle until the user can recover from the collapse event or contact the ground in a controlled manner that reduces the chance of injury. The participants ranged from 65 to $90 \mathrm{~kg}$ in weight, thereby covering a reasonable range of the end user population. Although nondisabled users could have attenuated knee flexion during the leg-collapse trials, participants did aggressively transfer all weight onto the braced leg after having had sufficient practice to be confident that the orthosis would safely stop knee flexion and prevent a fall. Therefore, the leg-collapse task was a reasonable approximation of a worst-case stumble.

The knee angle at which joint flexion resistance engaged during fast walking was approximately $25^{\circ}$. This is an interesting difference between the AVBC approach and commonly used stance-sensing approaches for kneeflexion control. Some end users may require a SCKAFO that limits the engagement angle to approximately $5^{\circ}$ to maintain a feeling of security when walking. For such cases, a posterior offset for the OWS AVBC joint may provide an appropriate combination of security during straight-leg limb loading and confidence that the orthosis is safe when landing with a flexed knee.

The maximum eccentric knee moments during leg collapse were larger than the mechanical test criterion (150 Nm), which was related to the maximum knee moments experienced during stair navigation [14]. The OWS successfully handled these knee moments and was able to provide appropriate knee moments to maintain the knee angle until double support was reinstated. Other SCKAFO joints that can engage at any angle had lower braking capacities; for example, $113 \mathrm{Nm}$ for the wrap spring clutch [20] and $69 \mathrm{Nm}$ for the belt-clamping [14] SCKAFOs.

Between cessation of knee flexion and collapse recovery, the linear hydraulic design maintained knee flexion within approximately $2^{\circ}$, which was a substantial improvement over the rotary hydraulic device that had $12^{\circ}$ of knee flexion during recovery [19]. While both hydraulic AVBC approaches provide sufficient time for recovery from a knee-collapse event, the linear hydraulic OWS may feel more secure with more rapid engagement of knee-flexion resistance. Since a lighter single-axis knee joint could be used on the medial side for a linear hydraulic OWS, device weight would be lower than a KAFO that required medial and lateral rotary hydraulic joints to support similar loads.

\section{CONCLUSIONS}

This study demonstrated the viability of a linear piston hydraulic orthosis for an AVBC approach of knee flexion. The OWS requires only a single lateral flexion-control joint to resist knee-collapse loading for the target user (maximum $90 \mathrm{~kg}$ ) and withstands more than the expected number of annual loading cycles. The OWS affected below-threshold walking gait in a similar manner as a standard single-axis KAFO. Fast walking speeds that caused the knee-flexion resistance to engage reduced the peak knee-flexion angle during initial swing but did not produce an unsafe condition. The OWS successfully engaged kneeflexion resistance and stopped knee flexion during a legcollapse event, enabling the study participants to safely regain a stable standing position. Following these positive outcomes, the OWS prototype design will be tested on assistive device users with knee-extensor weakness.

\section{ACKNOWLEDGMENTS}

\section{Author Contributions:}

Study concept and design: E. D. Lemaire, R. Samadi, L. Goudreau, J. Kofman.

Acquisition of data: R. Samadi.

Analysis and interpretation of data: E. D. Lemaire, R. Samadi, J. Kofman. Drafting of manuscript: E. D. Lemaire, J. Kofman.

Statistical analysis: E. D. Lemaire, J. Kofman.

Obtained funding: E. D. Lemaire, J. Kofman.

Financial Disclosures: The authors have declared that no competing interests exist.

Funding/Support: This material was based on work supported by the Canadian Institutes of Health Research (grant 78755) and the Natural Sciences and Engineering Research Council of Canada (grant 288232-2008).

Additional Contributions: The authors would like to acknowledge the clinical support from Ted Radstake and Rajiv Kalsi, technical support from Shawn Millar, and facilities support from The Ottawa Hospital Rehabilitation Centre.

Institutional Review: The Ottawa Hospital Research Ethics Board and University of Waterloo Office of Research Ethics reviewed and approved this project. All participants provided informed consent. Participant Follow-Up: The authors do not plan to inform participants of the publication of this study.

\section{REFERENCES}

1. Allard P, Duhaime M, Thiry PS, Drouin G. Use of gait simulation in the evaluation of a spring-loaded knee joint orthosis for Duchenne muscular dystrophy patients. Med Biol 
Eng Comput. 1981;19(2):165-70. [PMID:7266095] http://dx.doi.org/10.1007/BF02442710

2. Bowker P, Condie DN, Bader DL, Pratt DJ. Biomechanical basis of orthotic management. Oxford (England): Butterworth-Heinemann; 1993.

3. Drachlis D. Innovative knee brace moves closer to manufacturing with acceptance of final design. Marshall Space Flight Centre News Release, 098-032: 1998.

4. Fillauer [Internet]. Fillauer swing phase lock manual. Chattanooga (TN): Fillauer; 2012 [cited 2012 Jun 7]. Available from: http://www.fillauer.com/Orthotics/SPL2.html

5. Hebert JS, Liggins AB. Gait evaluation of an automatic stance-control knee orthosis in a patient with postpoliomyelitis. Arch Phys Med Rehabil. 2005;86(8):1676-80. [PMID:16084826] http://dx.doi.org/10.1016/j.apmr.2004.12.024

6. Irby SE, Bernhardt KA, Kaufman KR. Gait changes over time in stance control orthosis users. Prosthet Orthot Int. 2007;31(4):353-61. [PMID:17852777] http://dx.doi.org/10.1080/03093640601076909

7. Bernhardt KA, Irby SE, Kaufman KR. Consumer opinions of a stance control knee orthosis. Prosthet Orthot Int. 2006; 30(3):246-56. [PMID:17162515] http://dx.doi.org/10.1080/03093640600618818

8. Lehmann JF, Stonebridge JB. Knee lock device for knee ankle orthoses for spinal cord injured patients: an evaluation. Arch Phys Med Rehabil. 1978;59(5):207-11. [PMID:655831]

9. McMillan AG, Kendrick KK, Michael JW, Aronson J, Horton GW. Preliminary evidence for effectiveness of a stance control orthosis. J Prosthet Orthot. 2004;16(1):6-13. http://dx.doi.org/10.1097/00008526-200401000-00004

10. Moreno JC, Brunetti F, Rocon E, Pons JL. Immediate effects of a controllable knee ankle foot orthosis for functional compensation of gait in patients with proximal leg weakness. Med Biol Eng Comput. 2008;46(1):43-53. [PMID:17926076] http://dx.doi.org/10.1007/s11517-007-0267-x

11. Naft JM, Newman WS, inventors. Orthosis knee joint. United States patent US 6517503 B1. 2003 Feb 11.

12. Nijenbanning G, Goudsmit JA, inventors. Gravity operated locking hinge. United States patent US 6979304. 2005 Dec 27.

13. Van Leederdam NG, Kunst EE. Die neue beinorthese UTXSwing: normales gehen kombiniert mit sicherem stehen [New UTX-Swing orthosis: normal gait and safe standing]. Orthopadie Technik. 1999;50:506-15. Dutch.

14. Yakimovich T, Kofman J, Lemaire ED. Design and evaluation of a stance-control knee-ankle-foot orthosis knee joint. IEEE Trans Neural Syst Rehabil Eng. 2006;14(3):361-69.

\section{[PMID:17009496]}

http://dx.doi.org/10.1109/TNSRE.2006.881578

15. Yakimovich T, Lemaire ED, Kofman J. Preliminary kinematic evaluation of a new stance-control knee-ankle-foot orthosis. Clin Biomech (Bristol, Avon). 2006;21(10):1081-89. [PMID:16949186] http://dx.doi.org/10.1016/j.clinbiomech.2006.06.008

16. Zissimopoulos A, Fatone S, Gard SA. Biomechanical and energetic effects of a stance-control orthotic knee joint. J Rehabil Res Dev. 2007;44(4):503-13. [PMID:18247247] http://dx.doi.org/10.1682/JRRD.2006.09.0124

17. Carvajal F, Reilander K, Golkarieh H, Taebi S, Lemaire E. Power cost of an electronic control system for a stance control knee-ankle-foot orthosis. 30th Canadian Medical and Biological Engineering Conference; 2007 Jun 16-19; Toronto, Canada.

18. Kofman J, Allard P, Duhaime M, Labelle H, Vanasse M. A functional knee-ankle orthosis for Duchenne muscular dystrophy patients using a spring-loaded knee joint mechanism. Orthopadie Technik. 1985;36:403-7.

19. Lemaire ED, Goudreau L, Yakimovich T, Kofman J. Angular-velocity control approach for stance-control orthoses. IEEE Trans Neural Syst Rehabil Eng. 2009;17(5):497-503. [PMID:19497821] http://dx.doi.org/10.1109/TNSRE.2009.2023308

20. Irby SE, Bernhardt KA, Kaufman KR. Gait of stance control orthosis users: the dynamic knee brace system. Prosthet Orthot Int. 2005;29(3):269-82. [PMID:16466156] http://dx.doi.org/10.1080/03093640500238915

Submitted for publication February 17, 2012. Accepted in revised form June 4, 2012.

This article and any supplementary material should be cited as follows:

Lemaire ED, Samadi R, Goudreau L, Kofman J. Mechanical and biomechanical analysis of a linear piston design for angular-velocity-based orthotic control. J Rehabil Res Dev. 2013;50(1):43-52.

http://dx.doi.org/10.1682/JRRD.2012.02.0031

ResearcherID: Edward D. Lemaire, PhD: B-1502-2012

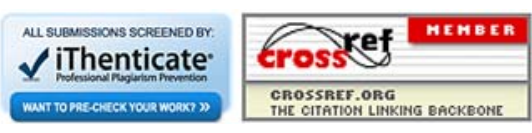

